\title{
Automata Semantics and Analysis of BPEL
}

\author{
Yongyan ZHENG, Paul KRAUSE \\ Department of Computing, University of Surrey \\ Guildford, GU2 7XH, UK \\ \{y.zheng, p.krause\}@surrey.ac.uk
}

\begin{abstract}
Web service is an emerging paradigm for distributed computing. In order to verify web services rigorously, it is important to provide a formal semantics for flow-based web service languages such as BPEL. A suitable formal model should cover most features of BPEL. The existing formal models either abstract from data, cover a simple subset of BPEL, or omit the interactions between BPEL activities. This paper presents Web Service Automata, an extension of Mealy machines, to fulfil the formal model requirements of the web service domain. Secondly, the paper analyses the control handling and data handling of BPEL, so that these can be verified in a clear manner.
\end{abstract}

Index Terms - Web service, BPEL, finite state machine, design model analysis.

\section{INTRODUCTION}

The web service paradigm provides a flexible, re-usable, and loosely coupled model for distributed computing. Web service architectures have been actively researched in recent years, and various web service standards have been proposed. BPEL [1] is one of the de-facto orchestration languages to model business processes as coordinated sets of web service interactions. Since BPEL is a semi-formal flow language, various formal semantics have been proposed, so that BPEL models can be verified rigorously. However, most current formal models do not cover the BPEL data flow analysis, and ignore the internal interactions of BPEL activities.

Existing model checking tools can be reused for the purpose of verification and testing of BPEL. Our formal model is intended to be used by such verification tools. With model checking, a BPEL model can not only be a design model for verification, but also be a test model for deriving test cases. The formal semantics proposed to date for BPEL can be categorized as process algebra based, Petri-net based, and automata based. We follow the automata-based approach, in order to facilitate the use of model checking tools. We propose a Web Service Automaton (WSA), an extension of Mealy machine, which covers data, supports message passing communication, and adapts the asynchronous interleaving semantics. We justify the suitability of WSA for BPEL on three counts. First, its propositional input events capture most features of the BPEL language, while most automata-based formal models for BPEL only cover the core subset features of BPEL. Second, its message passing communication provides a uniform semantics for both BPEL internal and external interactions. Third, the model analyzes BPEL control and data flows in separation.

The rest of the paper is organized as follows. Section II presents our web service automata semantics. Section III analyses BPEL data and control flows. Section IV reviews related work, and Section V concludes the paper.

\section{Web Service Automaton}

In the following, we give the formal definition for the static semantics and briefly describe the dynamic semantics of a web service automaton.

Definition 1. We assume that we have available a enumerable infinite set $V$ of variables and sets $A X, B Y$ of assignment expressions and Boolean expressions respectively, together with a set $D$ of values. We also assume that we have a set of functions $E n v$ where $\varepsilon \in E n v: V \rightarrow D$ assigns variable of $\mathrm{V}$ with a value from $D$. Given an expression exp: We need three functions:

- def: $A X \rightarrow V$, where def (exp) $\in V$ returns the assigned variable on the left hand side of the assignment.

- cuses: $A X \rightarrow \wp(V)$, where $\wp(V)$ the power is set of $V$ and cuses(exp) $\subseteq V$ returns the used variables on the right hand side of the assignment.

- puses: $B X \rightarrow \wp(V)$, where puses $(\exp ) \subseteq V$ returns the used variables in the Boolean expression.

Definition 2. A Web Service Automaton (WSA) $M$ is a finite state machine, consisting of $W S_{\mathrm{M}}=\left(I_{\mathrm{M}}, S_{\mathrm{M}}, S_{\mathrm{fM}}, T_{\mathrm{M}}, \delta_{\mathrm{M}}\right)$. As a convention, we omit the subscript of $M$ such that $M=\left(I, S, S_{\mathrm{f}}, T, \delta\right)$.

1) $I$ is the signature of $M$, denoted as a three tuple $I=(E, L, O)$, where $E, L, O$ are pair-wise disjoint and represent a set of input events, internal events, and output events, respectively. Let $M s g=(L \cup E \cup O)$ to be the set of events, we refer to the elements of $L_{\text {in }} \cup L_{\text {out }}$ as internal input and internal output events, and to those of internal events and to those of $E \cup O$ as external events.

2) $S$ is a set of states, $s_{0} \in S$ is the initial state, $S_{\mathrm{f}} \subseteq S$ is a set of final states.

3) $T \subseteq I N \times B X \times(\wp(A X) \cup O U T)$ is a set of transitions, where $I N=\left(E \cup L_{\text {in }} \cup\{\Omega\}\right)$ and $O U T=\left(L_{\text {out }} \cup O \cup\{\Omega\}\right)$. For each $t=(m, g, a) \in T$ (graphically denoted as $m[g] / a$ ), $m \subseteq I N$ is a triggering event, $g \in B X$ is the guard predicate, and $a \subseteq(\wp(A X) \cup O U T)$ is the action set composed of assignments and output events. Here $\Omega$ indicates the omission of either an event. We could repre- 
sent a transition by $\Omega[g] / \Omega$ which simply determines a state change and nothing else.

- The events of the transition input event set t. $m \subseteq I N$ are linked the by logical operation conjunction, disjunction, or negation, denoted as $A N D: e_{1} \wedge e_{2} . \wedge . . e_{\mathrm{n}}, O R: e_{1} \vee e_{2} . . \vee . . e_{\mathrm{n}}$, and NOT: $\neg e_{i}$, respectively.

- The data structure of machine $M$ is the form of $\left(V_{\mathrm{M}}, A X_{\mathrm{M}}, B X_{\mathrm{M}}\right)$, which can be retrieved from $T$.

4) $\delta \subseteq S \times T \times S$ is the transition relation.

For the dynamic semantics, the message-passing based asynchronous communication model is used in WSA. The machine composition adapts asynchronous interleaving semantics. A machine is associated with a multi-set buffer with FIFO queue for each message. We use symbols !,? as a convention in diagrams to indicate whether an event is input, output, denoted as $? e \in E$ and $! e \in O$, respectively.

\section{ANALYSIS OF BPEL FEATURES IN WSA}

BPEL consists of basic and structured activities. Basic activities are atomic actions. Structured activities impose control dependencies on the executions of either the basic or structured activities within them. A structured activity can contain an arbitrary depth of sub-activities. BPEL has structured activities including the pick, switch, while, sequence, flow, scope, eventHandlers, faultHandlers, and a compensationHandler structured activity to reverse completed activities. BPEL handles data using a blackboard approach, where a set of variables is shared by the enclosed activities.

In this section, we analyse the main features of BPEL and describe how to capture these features in WSA. The detailed mapping from BPEL to WSA can be found in [13]. We use machine as shorthand for a web service automaton, and call the machine associated with BPEL $\mathrm{x}$ activity as $\mathrm{x}$ machine. In state machine diagrams, an initial state is pointed by an arrow starting with a filled black circle, and a final state is shaded.

\section{A. Motivating Scenario}

We use a scenario to show the importance of analysing both BPEL control flow and data flow. In BPEL, variables and links may affect the control flow, variables may appear in expressions on the conditions in switch and while, and may also be used in the condition to fire particular links in the source element. So taking into account variables is essential in the formal model. There are two types of variables in BPEL: BPEL variables and links. BPEL variables are declared in the variables tag of either process or scope activity. Links are Boolean variables declared in the links tag of the flow activity. The output link of an activity is defined as true if the activity completes normally, otherwise the link is defined false. The link synchronization feature will be covered in subsection G. BPEL variables and links can be used and defined by the process or scope enclosed activities, and the flow enclosed activities, respectively.

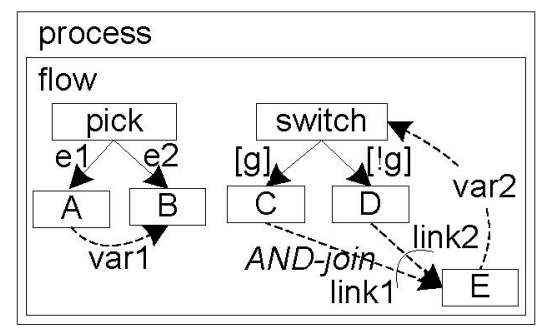

Fig.1 Unreachable and deadlock activities

In Fig. 1, the boxes, the solid arcs, and the dashed arcs denote BPEL activities, control flow, and data flow, respectively. The process activity encloses a flow, activity which in turn includes pick, switch, and $E$ activities running concurrently. The example contains unreachable and deadlock activities. Firstly, $B$ and $E$ are unreachable. $B$ is unreachable due to the interaction between data flow and control flow. In the pick, $A$ and $B$ are mutually exclusive in control flow, but the output of $A$ is the input of $B$ in data flow, so $B$ can never be executed. $E$ is unreachable due to the fault design of links. In the switch, $C$ and $D$ are mutually exclusive in control flow, so the link 1 and link 2 cannot be both true to satisfy the AND-join condition. Therefore, $E$ can never be executed. Secondly, there is a deadlock between switch and $E$, which is caused by the cyclic data flow between them. On the one hand, $E$ waits for both link 1 and link 2 to be true but this condition can never be satisfied. On the other hand, the switch waits for its input var2 to be defined but var2 cannot be defined by $E$ because of the falsity of either link1 or link2. This illustrates the necessity to verify both control flow and data flow.

\section{B. Loan Approval Example}

We use a loan approval process [1] as a running example to illustrate our data flow model and the control flow model, shown in Fig. 2. There exist three web services: loanapproval, assessor, and approver. A customer asks for a loan of a specified amount. Based on the request's amount, the loanapproval invokes either assessor or approver. If the assessor is invoked, based on the returned risk, the loanapproval either assigns the approval value itself or invokes the approver. The final approval value is relayed to the customer. The arrows represent message flow in this scenario diagram.

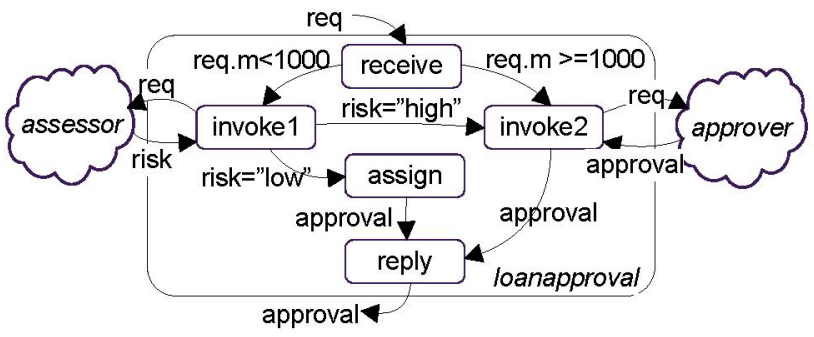

Fig. 2 Loan approval example

The BPEL model for the loanapproval service uses a process activity, flow activity and activities receive, invoke1, invoke2, assign, reply running concurrently within the flow. It has global variables request, risk, approval and 6 flow links to control which path the model will execute. 


\section{Control Flow Model}

A WSA has no hierarchy. We simulate the hierarchical relationships of BPEL activities by adding start message and done message as common administration messages between machines. A machine can play the role of parent or child. For a machine $M$, its parent machine is the one who sends a start message to $M$, while its child machine is the one who receives a start message from $M$. A child machine will send a done message to its parent machine when reaching one of its final states. If machine $M$ sends a start message to machine $N$, then $M$ is the parent machine of $N$ and $N$ is the child machine of $M$. A child machine will send a done message to its parent machine when reaching one of its final states. Each machine has zero or one parent machine, and zero or many children machines. Since the BPEL basic activity is atomic and a BPEL structured activity is hierarchi$\mathrm{cal}$, the machine for a BPEL basic activity has no child, and the machine for a BPEL structured activity has $0 . .^{*}$ children.

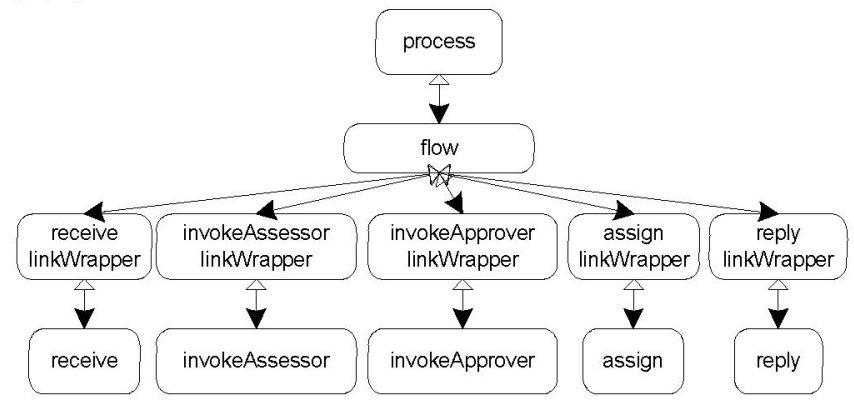

Fig.3 An example of machine control-flow model

Fig. 3 shows the machine control flow of the loan approval service. The machine without an incoming dark arrow (start message) is the process machine. The machine without an incoming hollow arrow (done message) is a $b a$ sic machine. The process machine is the parent of the flow machine, and the flow is the parent of receivelinkWrapper machine, which in turn is the parent of receive machine. In a flow activity, when an enclosed activity contains links, this activity will be associated with a core machine and a linkWrapper machine. The rationale for this is covered in subsection $\mathrm{G}$.

\section{Data Flow Model}

Data flow captures the relations between inputs and outputs of BPEL activities. In this paper, we focus on the data flow model of the internal BPEL activities. BPEL handles data by a blackboard approach, where data is shared by BPEL activities. By message passing, there are two possible ways to construct data flow from a BPEL model. One approach is to simulate the shared data access by adding data writing to and reading from the blackboard. The other approach is to analyse the BPEL model to discover data dependencies among activities. The data flows identified from these two approaches are called centralized and decentralized data flow, respectively. Fig. 4 shows the difference between them.
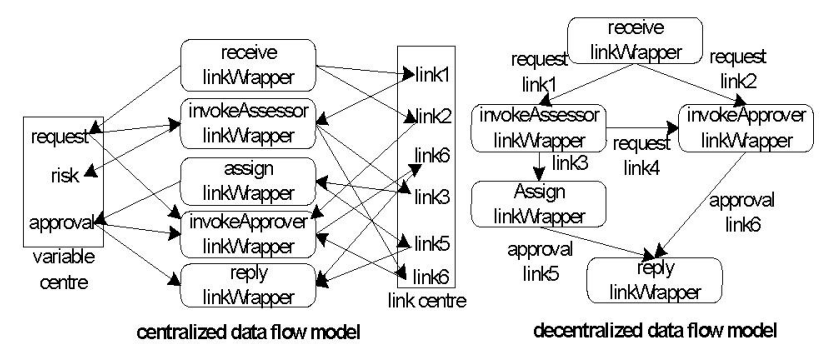

Fig.4 An example of machine data-flow models

In the centralized data-flow model, all the data exchanges between activities need to be via a variable or link centre. In the decentralized data-flow model, activities can exchange data directly. We choose the decentralized approach because it is more efficient in terms of data communication.

\section{E. Concurrency, Fault Propagation, and Interruption}

In this subsection, we show that a machine's input events with logical AND, OR, NOT can capture various BPEL features.

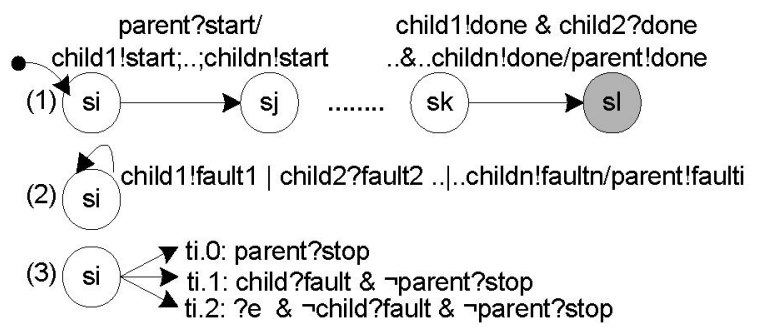

Fig.5 Propositional input events

\section{- Concurrency}

BPEL flow, scope, and eventHandler activities allow the enclosed activities to perform concurrently. We use flow activity as an illustration here. When the flow enters, all the enclosed activities start. The flow ends when all the enclosed activities end. We model this by two transitions, shown in (1) Fig. 5. On the left of (1), the flow machine starts all its children as a transition action, so that all child machines will start at the same time. On the right of (1), a logical-AND operator is added to the transition input events, so that the flow machine will not end until all its children end by sending done messages.

\section{- Fault propagation}

When a structured machine receives a fault message from its children, it forwards the fault message to its parent. Suppose the structured activity encloses more than one activity. The fault is propagated as long as one of the enclosed activities raises a fault. We model this by adding a logical-OR operator to the transition input events, shown in (2) of Fig. 5. Instead of using a queue for each fault, we use one FIFO queue to store all fault messages, so the fault message sent from the activity machine to its parent depends on which child's fault comes first. 


\section{- Interruptions}

BPEL has two kinds of Interruptions. First, when a termination message is thrown when a terminate activity is reached, the process machine ends abnormally, and a stop message is propagated downstream. Second, when a fault is thrown by a throw activity, the normal activity needs to stop before the fault handler starts. The fault is propagated upstream until it can be caught by a scope or process activity that has the fault handler for this fault. When a structured activity is stopped, all its children need to be stopped first. This is modelled by propagating a stop message downstream. The priority of a stop message is captured by adding logical-AND together with logical-NOT to transition input events. A stop message has higher priority than a fault message, which in turn has higher priority than a normal message. In (3) of Fig. 5, transition $t_{i .0}$ is triggered when either a stop or a term message arrives. The $t_{\mathrm{i} .1}$ will be triggered when it does not receive a fault message from its child, and only when neither stop nor term message arrives. It indicates that a fault will not be propagated when the machine is asked to stop or terminate. The $t_{1.2}$ indicates that a fault or interruption message has higher priority than a normal incoming message.

\section{F. Common structured machine layout}

With consideration to fault propagation and machine interruption, BPEL structured activities have a common machine layout, shown in Fig. 6.

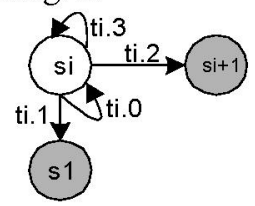

Fig.6 The common structured machine layout

Each structured activity machine has a stopStatus local variable. When the machine receives the children's done messages, based on the value of stopStatus, the machine enters a normal or abnormal final state. Suppose $M$ is a structured machine, we can derive three scenarios from the common machine structure: 1) when $M$ receives the children's done messages and the stopStaus is false $\left(t_{1.2}\right)$, it ends at normal final state $s_{i+1} ; 2$ ) when $M$ receives a stop message from its parent, it propagates the message to its children $\left(t_{i .0}\right)$ and update the stopStatus to true. Given the true value of stopStatus, $M$ enters the abnormal final state $s_{1}$ after receiving its children's done messages $\left(t_{\mathrm{i} .1}\right) ; 3$ ) when $M$ receives a fault from its children, it and forwards the message to its parent $\left(t_{1.3}\right)$, and follows the 2) scenario.

\section{G. Synchronization of Concurrent Activities and Dead- Path-Elimination}

A set of links can be declared in the flow construct to express the synchronization dependencies between activities within a flow. A link is a Boolean variable, and each link is associated with a pair of source activity and target activity. For instance, if $M$ and $N$ are source and target activities of link1, respectively, link1 is $M$ 's outgoing link with source tag, and $N$ 's incoming link with target tag.

The synchronization between source and target activities is realized by setting and getting the link value. The source activity sets the link to be true or false, and the target activity gets the link value. The target activity can start when 1) all the incoming links' values are defined by the source activities, and 2) its associated join-condition is satisfied, which is either an AND or OR logical constraint on link values. If the join-condition is false, the target activity will not be executed and this effect will be propagated downstream in the flow model. This is called Dead-PathElimination in BPEL. We capture the dead-path-elimination feature by updating the related links to false, and sending the setLink messages to the target machines.

The target tag and source tag are standard elements of BPEL constructs, indicating each BPEL activity may or may not have incoming links and outgoing links. To capture this feature, we use a separate linkWrapper machine to handle links. When an activity has incoming or outgoing links, it will associate with a linkWrapper machine and a core machine. The linkWrapper will be the core machine's parent. When an activity has no link, it is only associated with a core machine. This separation simplifies the structure of a machine, and allows BPEL activities to share a common machine structure for link handling.

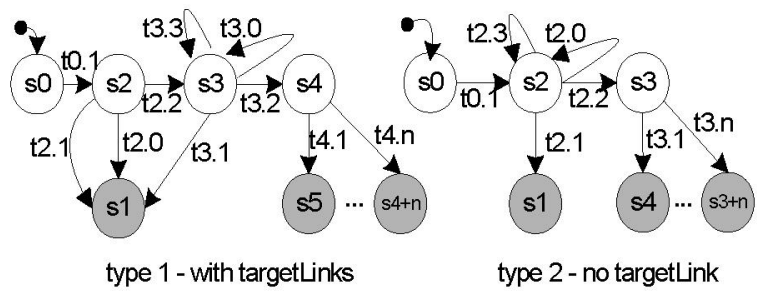

Fig. 7 The linkWrapper machines

Fig. 7 shows the two types of linkWrapper machine structures, which covers the cases when an activity has target links and no target link. We use the type 1 linkWrapper structure as an illustration. Suppose $B$ is the core machine and $A$ is the linkWrapper machine for an activity, several scenarios can be derived from the machine structure.

First, a normal scenario follows the path $\left\langle t_{0.1}, t_{2.2}, t_{3.2}, t_{4 . x}\right\rangle$. Machine $A$ starts by receiving a start message from its parent $t_{0.1}$. It receives target link values from the link's source machine, and if the links' values satisfy the join-condition, it starts $B\left(t_{2.2}\right)$. Machine $A$ sends its done message to its parent after receiving $B$ 's done message $\left(t_{3.2}\right)$. If $x$ and $y$ are $B$ 's input and output, respectively, then $A$ will send $x$ to $B$ and receive $y$ from $B$. Thereafter, machine $A$ sets the corresponding source link to true and the other source links to false, and sends the setlink messages to the links' target machines $\left(t_{4 . x}\right)$. Each outgoing transition of state $s_{4}$ corresponds to a source link.

Second, alternative scenarios can be: 1) $A$ is interrupted by a stop message from its parent $\left(t_{2.0}\right) ; 2$ ) when the joincondition is false, $A$ updates all the source links to false and sends them to the links' target machines, and $A$ ends abnor- 
mally $\left.\left(t_{2.1}\right) ; 3\right)$ when $A$ is interrupted by a stop message from its parent after $B$ started, it stops $B\left(t_{3.0}\right)$. After receiving $B$ 's done message, $A$ sends a done message to its parent $\left(t_{3.1}\right) ; 4$ ) when $A$ receives fault from $B$, it forwards the fault to its parent $\left(t_{3.3}\right)$ and follows scenario 3$)$.

\section{H. Scope, Compensation and Fault Handling}

A scope has a primary activity that defines its normal behaviour, and it can optionally enclose eventHandlers (EHS), faultHandlers $(\mathrm{FH})$, and compensationHandler $(\mathrm{CH})$ activities. In a scope, EHS runs concurrently with a primary activity. The right of Fig. 8 shows the hierarchy of invoking a $\mathrm{CH}$. Only $\mathrm{CH}$ and $\mathrm{FH}$ are allowed to send a compensate message, and the target machine of this message must be a scope (scope2) immediately enclosed in the current scope (scope 1). When receiving a compensation message, scope 2 starts its $\mathrm{CH}$ to do the compensation.

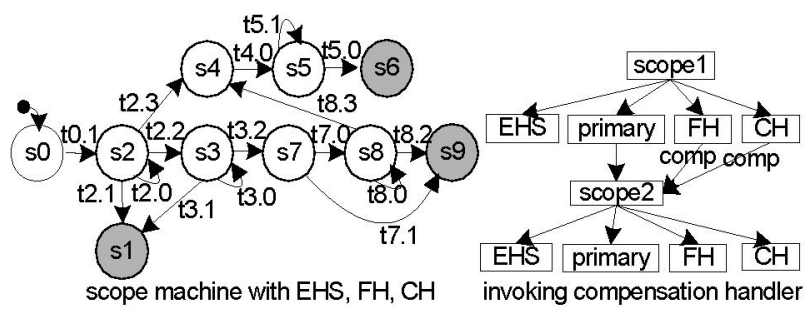

Fig. 8 Scope and Compensation

The left of Fig. 8 shows a scope machine with EHS, FH, and $\mathrm{CH}$. When the scope is started, it starts the primary machine and EHS $\left(t_{0.1}\right)$, it waits for the primary machine to finish at state $s_{3}$. When it receives primary machine's done message, it disables EHS $\left(t_{2.2}\right)$. The machine enters $s_{7}$ when the EHS is done message $\left(t_{3.2}\right)$. If the scope does not receive a compensate message when its EHS ends at $s_{7}$, the execution path is $\left\langle t_{0.1}, t_{2.2}, t_{3.2}, t_{7.1}\right\rangle$. Otherwise, the execution path is $\left\langle t_{0.1}, t_{2.2}, t_{3.2}, t_{7.0}, t_{8.2}\right\rangle$. A scope's $\mathrm{CH}$ is available for invocation only when the scope completes normally. We model this by $t_{7.0}$ and $t_{8.2}$. When the scope receives a compensate message, it starts its $\mathrm{CH}\left(t_{7.0}\right)$. The scope is done after the $\mathrm{CH}$ is done $\left(t_{8.2}\right)$.

Alternatively, when the scope receives a fault, it stops its children and starts the $\mathrm{FH}\left(t_{2.3}\right)$. When the primary machine and the EHS finish, it enables the FH $\left(t_{4.0}\right)$. The scope may receive a fault re-throw from the $\mathrm{FH}$, and it forwards the fault to its parent $\left(t_{5.1}\right)$. The scope ends when the FH finishes $\left(t_{5.0}\right)$. When the scope is interrupted to stop, the implicit 'bpws:forceTermination' faultHandler will run. For simplicity, we do not model this implicit faultHandler and instead we use the stop message to stop its children, and the common machine structure for BPEL structured activities is also used in scope.

\section{Multiple Threads of Message Event Handlers}

The eventHandlers activity consists of a set of concurrent activities onMessage and onAlarm activities. The onMessage and onAlarm activities handle external message events and system alarm events, respectively. An alarm event is carried out at most once, while a message event can occur multiple times when the scope is active. We model this by associating the eventHandlers activity with EHS machine, each onMessage activity with a message event handler machine (MEH), each onAlarm activity with an alarm event handler machine $(\mathrm{AEH})$, and each thread of onMessage activity with a $\mathrm{MEH}$ thread machine (MEHT). Each thread takes care of one message instance.
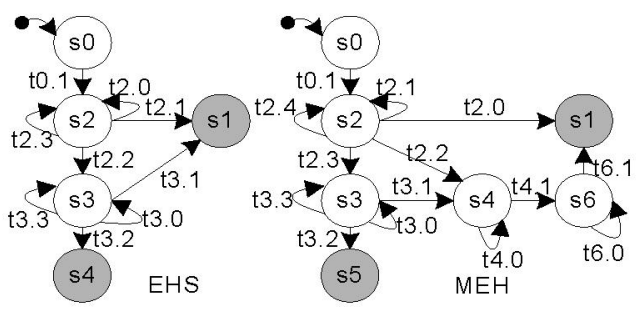

Fig.9 EHS and MEH machines

Fig. 9 shows the EHS, MEH, and MEHT machines. For the EHS machine, we only show the normal scenario for simplicity. The parent of EHS is a scope machine and the children of EHS are MEHs and AEHs. When receiving a start message from the scope, EHS starts its children $\left(t_{0.1}\right)$. The EHS will not end until it receives a disable message from the scope. When EHS receives a disable message, it forwards the message to its children $\left(t_{2.2}\right)$. When EHS receives its children's done messages, it sends a done message to its parent $\left(t_{3.2}\right)$.

For the MEH machine, since a MEH may start a thread for each message event instance, and the thread number is unknown, we model this by adding local variables count for the current thread number.

The normal scenario of $\mathrm{MEH}$ is $\left\langle t_{0.1}, t_{2.1}, t_{2.3}, t_{3.0}, t_{3.2}\right\rangle$. When it receives a start message from the parent, the count is initiated to zero $\left(t_{0.1}\right)$. When it receives an external message, it increases the Itextit\{count\} by 1 and starts a new thread as a child machine $\left(t_{2.1}\right)$. The machines waits for an external message event to arrive at $\mathrm{s} 2$, a new thread is created for each message instance $\left(t_{2.1}\right)$. When it receives a disable message from its parent and the count is not zero, it enters state $s_{3}$ to wait for its child machine to finish $\left(t_{2.3}\right)$. When one of its children finishes, it decreases the count by 1 , until all the children finish $\left(t_{3.0}\right)$. When the count is zero, $\mathrm{MEH}$ ends at a normal final state $\left(t_{3.2}\right)$.

An alternative scenario is $\left\langle t_{0.1}, t_{2.0}\right\rangle$ where the machine is disabled before starting any thread. The other two alternative scenarios contain transition sequence $\left\langle t_{4.0}, t_{4.1}, t_{6.0}, t_{6.1}\right\rangle$, when the machine is interrupted by a stop message $\left(t_{2.2}, t_{3.1}\right)$, it stops its child machine one by one until all the children have been stopped $\left(t_{4.0}\right)$, then it enters state $s_{6}$ to wait for its children to finish $\left(t_{4.1}\right)$. Similar to $t_{3.0}$ and $t_{3.2}$, when one of its children finishes, it decreases the count by 1 until all the children finish $\left(t_{6.0}\right)$. MEH ends at a normal final state $\left(t_{6.1}\right)$.

\section{RELATED WORK}

In the literature, there are a number of proposals for 
BPEL formal semantics, such as process algebras, petri nets, and automata. $[8,10]$ give good reviews of the existing web services techniques. Ferrara [4] uses the process algebra LOTOS as the formal model and toolbox CADP is used as the verification engine. In LOTOS, rendezvous is used for the process communication model, and the disabling operator is used to capture the BPEL interruptions. Xu [12] applies process algebra Pi-calculus as the formal model and NuSMV model checker as the verification engine. The process algebra approaches cover most BPEL features. Wombacher [11] maps BPEL into deterministic finite state automata for the matchmaking of web service composition. Foster [5] uses FSPs (Finite State Processes) as the BPEL formal semantics. Both formalisms abstract from data. $\mathrm{Fu}$ and Bultan [6] propose guarded automata (GA) with data and abstract from internal BPEL activity, and use the SPIN model checker as the verification engine. Their GA only covers a core subset of BPEL, and they omit the interactions between internal BPEL activities.

Process algebra approaches have the advantage that the composition operators of process algebras are convenient in capturing the semantics of BPEL structured activities, and they support simulation and bi-simulation analysis, which are useful for model substitution and refinement. However, for the purpose of verification and testing, the automaton formalism is especially attractive due to the straight usage of model checking tools. The model substitution and refinement is not the focus of our test framework. Therefore, we have investigated the usability of automata approaches. We summarize our differences from the existing works as follows. 1) WSA supports propositional input events, which can reduce unnecessary machine state space and can capture most BPEL features. Most automata based approaches only cover a core subset of BPEL. 2) WSA captures both BPEL control flow and data flow explicitly, so that either control or date related errors in BPEL can be verified. 3) Most approaches do not consider the interactions between internal BPEL activities explicitly, but leave them to interact implicitly by shared variables. In the theoretical point of view, we believe it is clearer and simpler to provide a uniform means of interaction, instead of considering both shared variable and message passing mechanisms.

\section{Conclusion and Future Work}

We present Web Service Automata as a formal semantics for web services, and analyse various features of BPEL. The web service automaton is more general than the existing automata-based semantics in that it can model most features of BPEL and it allows verification of BPEL control and data flows. We encoded the web service automata into XML files. Also, we implemented a front end mapping from BPEL to web service automata, and the back end mapping from web service automata to the input languages of NuSMV and SPIN model checkers [2,7]. Based on test coverage criteria such as state coverage and transition coverage for control flow, and du-path coverage for data flow, model checkers can generate counter-examples. The test cases can be extracted from the counterexamples. This test generation framework was developed as an Eclipse plug-in [14], and it is part of the DBEStudio delivery for the EU project [3].

An open issue is to ensure the correctness of formal models, i.e. preservation of the BPEL semantics. We can partially verify the formal models, by defining some BPEL features as system properties in temporal logic and model check the formal models against these properties. An extension of this work is to apply our formal model to a choreography language such as WS-CDL[9]. We believe the same approach can be used due to the similar features of WSCDL and BPEL.

\section{ACKNOWLEDGMent}

This work is supported by the EU FP6 funded project Digital Business Ecosystem (project No. 507953).

\section{REFERENCES}

[1] T. Andrews, F. Curbera, H. Dholakia, Y. Goland, J. Klein, F. Leymann, K. Liu, D. Roller, S. Thatte, and S. Weerawarana. Business process execution language for web services version 1.1. May 2003.

[2] A. Cimatti, E. M. Clarke, F. Giunchiglia, and M. Roveri, "Nusmv:a new symbolic model verifier," in Proc. of CAV. Springer-Verlag, 1999 , p. 495.

[3] Digital Business Ecosystems (DBE)., available: http://www.digitalecosystem.org.

[4] A. Ferrara, "Web services:a process algebra approach," in Proc. Of ICSOC. ACM Press, 2004, p. 242.

[5] H. Foster, S. Uchitel, J. Magee, and J. Kramer, "Model-based analysis of obligations in web service choreography," in Proc. of AICT-ICIW. IEEE Computer Society, 2006, p. 149.

[6] X. Fu, T. Bultan, and J. Su, "Synchronizability of conversations among web services," IEEE Transactions on Software Engineering, vol. 31, no. 12, pp. 1042-1055, 2005

[7] G. J. Holzmann, The SPIN Model Checker: Primer and Reference Manual. Addison-Wesley Professional, 2003

[8] R. Hull and J. Su, "Tools for design of composite web services," in Proc. of SIGMOD. ACM Press, 2004, p. 958.

[9] N. Kavantzas, D. Burdett, and G. Ritzinger. Web services choreography description language version 1.0. April 2004.

[10] N. Milanovic and M. Malek, "Current solutions for web service composition," IEEE Internet Computing, vol. 08, no. 6, pp. 51-59, 2004.

[11] A. Wombacher, P. Fankhauser, and E. Neuhold, "Transforming bpel into annotated deterministic finite state automata for service discovery," in Proc. of ICWS. IEEE Computer Society, 2004, p. 316.

[12] K. Xu, Y. Liu, and G. Pu, "Formalization, verification and restructuring of bpel models with pi calculus and model checking," IBM, Technical Rep., 2006.

[13] Y. Zheng and P. Krause, "An automatic test generation framework for bpel web services," University of Surrey, Technical Rep., 2006.

[14] Y. Zheng and P. Krause, "A model checking based test case generation framework for web services," in Proc. of ITNG. IEEE Computer Society, 2007. 